\title{
(Q) Equity effects of parenting interventions for child conduct problems: a pan-European individual participant data meta-analysis
}

\begin{abstract}
Frances Gardner, Patty Leijten, Victoria Harris, Joanna Mann, Judy Hutchings, Jennifer Beecham, Eva-Maria Bonin, Vashti Berry, Sinead McGilloway, Maria Gaspar, Maria João Seabra-Santos, Bram Orobio de Castro, Ankie Menting, Margiad Williams, Ulf Axberg, Willy-Tore Morch, Stephen Scott*, Sabine Landau*
\end{abstract}

\section{Summary}

Lancet Psychiatry 2019; 6: 518-27

Published Online May 6, 2019

http://dx.doi.org/10.1016/ S2215-0366(19)30162-2 See Comment page 458 *Both authors contributed equally

Social Policy and Intervention, University of Oxford, Oxford, UK (Prof F Gardner DPhil, P Leijten PhD, J Mann PhD); University of Amsterdam, Amsterdam, Netherlands (P Leijten); Department of Biostatistics (V Harris PhD, Prof S Landau PhD), and Department of Child and Adolescent Psychiatry (Prof S Scott FRCPsych), King's College London, London, UK; Department of Psychology Bangor University, Bangor, UK (Prof) Hutchings DClinPsy, M Williams PhD); Personal Social Services Research Unit, London School of Economics, London, UK (Prof) Beecham PhD,

E-M Bonin PhD); Institute for Health Services Research, University of Exeter, Exeter, UK

(V Berry PhD); Department of Psychology, Maynooth

University, Maynooth, Ireland (Prof S McGilloway PhD); Psicologia e Ciências da Educação, University of Coimbra, Coimbra, Portugal (M Gaspar PhD,

M João Seabra-Santos PhD); Department of Developmental Psychology, Utrecht University, Utrecht, Netherlands (Prof B Orobio de Castro PhD, A Menting PhD); Department of Psychology, University of Gothenburg, Gothenburg, Sweden (U Axberg PhD); and Faculty of Health Science,

University of Tromsø, Tromsø, Norway (W-T Morch PhD)

Background Childhood conduct problems are a costly public health problem and are five times more common in socially disadvantaged groups than they are in advantaged groups. Untreated, conduct problems have a poor prognosis, with increasing gaps between socioeconomic groups, and high rates of subsequent criminality. Incredible Years is a high quality parenting programme for reducing conduct problems and is widely disseminated in Europe. Many trials have shown Incredible Years to be effective but the potential effects of parenting interventions on social inequality are unknown. Some behavioural interventions (eg, smoking cessation programmes), although beneficial overall, can widen inequality gaps. Because single trials and aggregate-level meta-analyses are ill equipped for examining differential intervention (moderator) effects, we pooled individual-level trial data to assess the effects of Incredible Years on social equity.

Methods We did a systematic review and individual participant data meta-analysis by searching CINAHL, Embase, Global Health, Medline, and PsycINFO, for studies published from inception to March 15, 2019. We also searched the Incredible Years website library and consulted with experts, including the European Incredible Years mentors' network. We included data from all completed randomised trials of the Incredible Years parenting intervention in Europe that included children aged 1-12 years, including unpublished trials, without restriction on publication year or outcome measures. We included prevention (selective or universal) and treatment or indicated prevention trials (for children diagnosed or above the clinical cutoff for conduct problems). We excluded trials or conditions within trials that were not randomised, included additional non-parenting material (eg, child-focused interventions), or were abbreviated, non-standard versions of the usual Incredible Years intervention of 12-14 weekly sessions. We requested individual participant data from the study authors. The primary outcome was child conduct problems, assessed using the Eyberg Child Behavior Inventory Intensity (ECBI-I) scale. Moderators were analysed using multilevel modelling with multiple imputation.

Findings Of 15 European trials of Incredible Years parenting programmes ( $n=1696$ children), individual participant data were unavailable for one trial and one trial did not assess the primary outcome. Children were aged 2-10 years (median 5 1), 492 (30\%) of 1651 children were from an ethnic minority and 931 (58\%) of 1614 were from low-income families. Families who received the Incredible Years intervention reported an overall reduction in child conduct problems (13.5 points on the ECBI-I scale, 95\% CI 10.9-16.1). There were no differential effects by family disadvantage (indicated by poverty, lone parenthood, teenage parenthood, household joblessness, or low education), or ethnic minority status.

Interpretation We found no evidence for differential effects by social disadvantage, suggesting that Incredible Years is unlikely to widen socioeconomic inequalities in conduct problems. Furthermore, the programme might be an important tool for reducing social disparities and improving poor long-term outcomes in disadvantaged families because follow-up studies indicate that benefits persist. Clinicians and commissioners can be reassured that the programme is similarly effective for families from different backgrounds.

Funding UK National Institute for Health Research.

Copyright (C) 2019 Elsevier Ltd. All rights reserved.

\section{Introduction}

Conduct problems in childhood are common, costly, and persistent over time, predicting many adverse outcomes, including poor school outcomes, criminal offending, unemployment, and poor physical and mental health. ${ }^{1,2}$
All such outcomes carry substantial economic and personal burden ${ }^{2}$ and are of major societal concern. Parenting interventions are a common and effective strategy ${ }^{3,4}$ for preventing and treating conduct problems, which also reduce harsh and abusive parenting. The past 


\section{Research in context}

\section{Evidence before this study}

Childhood conduct problems (disruptive behaviour problems) are a costly public health problem that is five times more common in socially disadvantaged groups than in advantaged groups. Incredible Years is a high-quality parenting programme for reducing child conduct problems and is widely disseminated across Europe. Some behavioural interventions, while beneficial on average, can often widen health gaps between rich and poor people (eg, smoking cessation programmes). Many trials and systematic reviews have shown Incredible Years to be effective but the potential effects on social inequality of parenting interventions are unknown. We systematically searched CINAHL, Embase, Global Health, Medline, and PsycINFO for previous systematic reviews of differential effects of parenting interventions published from inception to Dec 31, 2014, with no language restriction, using the search terms "parenting", "children" "conduct problems", and synonyms. We found two meta-analyses from 2006, addressing the moderation of parenting intervention effects by social disadvantage on the basis of aggregate-level data from randomised and non-randomised trials. These studies concluded that socially disadvantaged families benefited less from parenting interventions than did advantaged families. However, aggregate-level meta-analysis is a poor quality method for testing intervention moderation effects because of problems of low power, increased risk of bias, and absence of information about variability in outcome between individuals. To overcome these limitations, an individual participant data (IPD) meta-analysis is recommended. We found no IPD meta-analyses of parenting intervention trials in children.

\section{Added value of this study}

We did the first IPD meta-analysis of a parenting intervention, using individual-level data from almost all trials across Europe of Incredible Years. Our study is the first to test whether parenting programmes widen social inequalities in children's conduct problems, in a large, well-powered study. We analysed data from a near-complete set of eligible randomised trials of Incredible Years parenting programmes in Europe. A wide range of social and ethnic backgrounds and community-based service contexts were represented in the pooled sample. We found that Incredible Years was effective for reducing child conduct problems and that there was no evidence to suggest that the programme's effectiveness varied with different levels of social disadvantage, including for families on low income, lone or teenage parents, jobless households, or families with low education, as well as for ethnic minority families.

\section{Implications of all the available evidence}

Our findings suggest that this parenting programme to reduce childhood conduct problems, unlike many behavioural interventions for conditions that are considerably more common in disadvantaged groups, is unlikely to widen inequalities, based on socioeconomic status, or ethnicity. Our evidence updates and substantially alters the picture of inequality effects found in previous aggregate-level meta-analyses that have been influential. Furthermore, if the effects are maintained over time (initial studies suggest that they are), then the programme might reduce the increasing social gap in untreated conduct problems and the social difficulties and criminality that otherwise can ensue. Incredible Years could therefore be an important tool for promoting equity and clinicians, social care providers, and commissioners can be reassured that the programme is similarly effective for families from different backgrounds.
10-15 years have seen policy directives encouraging their implementation at scale in many European countries, and globally (eg, National Institute for Health and Care Excellence and WHO)., ${ }^{5,6}$

However, in going to scale, not only is establishing that interventions are effective important, but also that they are beneficial for the most socially disadvantaged, who typically have the poorest health. Poor parenting and child conduct problems are highly patterned by socioeconomic status, and possibly by ethnicity. ${ }^{7.8}$ A systematic review ${ }^{9}$ suggested that some types of behavioural interventionseg, for smoking cessation or obesity-although effective overall, might actually increase health inequalities, especially those that involve individual behavioural change, compared with those using external incentives or regulatory strategies. ${ }^{9}$ Therefore, the assessment of whether behavioural change interventions, including parenting programmes, have the unintended consequence of widening social inequalities in health is crucial, especially given widespread use of such interventions.
This question is key for commissioners and practitioners who refer families to interventions or deliver interventions to disadvantaged or minority client groups.

Despite substantial evidence that parenting interventions are effective overall in preventing and treating child conduct problems, ${ }^{3,4}$ there are reasons why disadvantaged families might benefit less than advantaged families. For example, economic stressors present barriers to attending sessions and implementing new strategies. Furthermore, programme developers might have a poor understanding of the child-rearing contexts and values of disadvantaged families, leading to a programme design that is better suited to middle-class families. Conflicting findings from individual trials and systematic reviews are equivocal about differential effects for socially disadvantaged or ethnic minority families..$^{10-13}$ For example, two systematic reviews addressing moderator effects concluded that disadvantaged children benefit less than do those from more advantaged families. $^{12,13}$ If this finding were true, parenting
Correspondence to:

Dr Frances Gardner, Social Policy and Intervention, Oxford University, Oxford, OX1 2ER, UK frances.gardner@spi.ox.ac.uk 
interventions might not help the most at-risk families, while inadvertently widening social inequalities. Such unintended effects were seen in the use of Sure Start community services in England, whereby the most socially disadvantaged families had worse outcomes compared with more advantaged families. ${ }^{14}$

Parenting styles, practices, and norms vary across cultures, but most parenting interventions are developed in high-income countries. Thus, interventions might be a poor fit and produce diminished effects for parents from different cultural backgrounds to those of the developers. Identifying effective cross-cultural parenting interventions is of huge importance to governments seeking to enhance health outcomes for minority ethnic and new immigrant families. Furthermore, worldwide policies promoting early intervention have led to considerable efforts to identify effective parenting interventions across cultures. ${ }^{5,15}$ Thus, understanding the effects of parenting interventions for minority families in high-income countries has important policy and practice implications, while contributing useful knowledge on the generalisability of interventions developed (and often delivered) by professionals from one ethnic background.

Most work on ethnicity and parenting interventions has been done in the USA, and is equivocal about whether outcomes differ by ethnicity. ${ }^{16,17}$ Despite this ambiguity, a predominant view exists that parenting interventions should be adapted for various ethnic groups. ${ }^{18}$ However, such approaches imply delivering interventions to parenting groups separately by ethnicity, which might be neither a practical nor desirable service model for multiethnic European cities. Moreover, data are needed to guide policy in Europe, where immigration has increased, but where individual trials are not powered to test intervention effects by ethnicity.

Traditionally, differential intervention effects are tested using subgroup analyses within trials or an aggregatelevel meta-analysis across trials. However, the validity of both traditional methods is insufficient; subgroup analyses in individual trials have well documented problems of inadequate power, multiple testing, and selective reporting bias, linked to failure to prespecify secondary analyses. ${ }^{19,20}$ Aggregate-level meta-analyses do not address these problems because moderators are analysed only at a trial level (eg, mean poverty level of the sample), leading to substantial loss of power and meaningful information, and creating a high risk of confounding moderators (eg, where poverty is correlated with problem severity). ${ }^{19,20}$

An individual participant data (IPD) meta-analysis helps to overcome these problems. First, the analysis fully exploits within-trial variation in sociodemographic characteristics, rather than lacking this information as in traditional meta-regression. ${ }^{20}$ Second, pooling across multiple trials allows for the inclusion of data on all outcomes and consistent analysis across trials, which maximises transparency and minimises bias, particularly selective outcome reporting and publication bias. However, benefits of IPD meta-analysis that reduce bias apply only if a large proportion of triallists share data and measure similar outcomes. Thus, IPD offers substantial advantages for addressing questions about equity effects, provided sufficient data can be included..$^{20}$ Equity questions are particularly pressing for problems that are highly patterned by social disadvantage, as with conduct problems, whose sequelae also confer further disadvantage. ${ }^{2}$

We aimed to assess, using IPD meta-analysis, the equity effects - in a European context - of the Incredible Years programme (appendix), a parenting intervention that is well established, group based, and evidence based for reducing child conduct problems. ${ }^{21}$ Specifically, we examined whether social disadvantage and ethnicity moderated the effects of Incredible Years intervention on conduct problems, using IPD across 13 European randomised trials.

We focused on the Incredible Years programme because: (1) it is a manualised intervention with a substantial evidence base, ${ }^{21}$ as recommended by NICE and other policymakers; (2) the intervention has been widely disseminated in many European countries; and (3) active European research networks exist for the programme, raising the probability of obtaining data from a near-complete set of trials for IPD meta-analysis. We focused on Europe because European countries where Incredible Years is implemented tend to have fairly similar health and social care systems (different from, for example, those in the USA), most European trials have been done independently of the programme developer (developer involvement is associated with increased intervention effects, and might represent a source of bias), and little is known about ethnicity effects in Europe, so pooling IPD allowed for the inclusion of substantial numbers of families from a range of ethnic minority backgrounds.

\section{Methods \\ Search strategy and selection criteria}

In January, 2015, we did a systematic review by searching CINAHL, Embase, Global Health, Medline, and PsycINFO, for studies published from inception to Dec 31, 2014. We also searched the Incredible Years website library and consulted with experts, including the European Incredible Years mentors' network (figure 1, appendix). Search terms via OVID were "incredible years.mp", "webster-stratton. $\mathrm{mp}$ ", and "incredible years.mp OR webster-stratton.mp". Search strings were adapted for other databases. Eligibility was assessed by the first author and double-checked by four additional authors, with no disagreements. We updated the search on March 15, 2019, to assess any relevant new trials. We followed PRISMA IPD reporting guidelines (appendix). Ethics approval was granted by Oxford University, Social Policy and Intervention Departmental Research Ethics Committee. 
We included all data from all completed randomised trials of the Incredible Years parenting intervention in Europe that included children aged 1-12 years, including unpublished trials, without restriction on publication year or outcome measures. We included prevention (selective or universal) and treatment or indicated prevention trials (for children diagnosed or above the clinical cutoff for conduct problems). We excluded trials or conditions within trials that were not randomised, included additional non-parenting material (eg, childfocused interventions), or were abbreviated, non-standard versions of the usual Incredible Years intervention of 12-14 weekly sessions.

All fully anonymised data that were available were requested for all randomised trials of the Incredible Years parenting intervention (table 1) identified in the 2015 searches. Trial principal investigators signed datasharing agreements specifying ethical and ownership issues. Raw, individual, item-level data were supplied and checked for missing items, scale validity and scores, internal consistency, baseline imbalance, and consistency with trial protocols and reports. Copies of original questionnaires were requested to check for consistent use across trials. Queries were resolved in collaboration with trial investigators. No substantial integrity issues arose. Trial quality was assessed using the Cochrane riskof-bias tool.

\section{Data analysis}

The prespecified primary outcome was the Eyberg Child Behavior Inventory Intensity (ECBI-I) score $^{22}$ because it is the most frequently used scale across trials. This 36-item scale is psychometrically robust and assesses parent-reported frequency of child conduct problems on a 7-point Likert scale.22 Two trials ${ }^{23,24}$ used a different measure of conduct problems (Parental Account of Children's Symptoms [PACS] $)^{25}$ and, in both cases, data were converted to scores on the ECBI-I, using norm deviation scores (appendix). This method is similar to using $Z$ scores, but uses means and standard deviations on each measure, from published norms. ${ }^{26,27}$

Given that social disadvantage is multifaceted, we included a range of indicators. Because of variation in the assessment of social disadvantage, we harmonised indicators on the basis of similarities in operationalisation. Indicators of low income were defined as receiving income-related financial benefits (ten trials), scoring below the Hollingshead Index's low-socioeconomic threshold (one trial), ${ }^{32}$ or living in social housing (two trials). ${ }^{33,34}$ The highest educational level of the primary parent was dichotomised using categories from UNESCO's international standard classification of education 2011,35 in which low means completed primary or lower secondary education, and high means completed upper secondary or degree-level education. Lone parenthood was indicated by whether the primary parent lives without a partner or spouse. Teenage parenthood was indicated by whether the

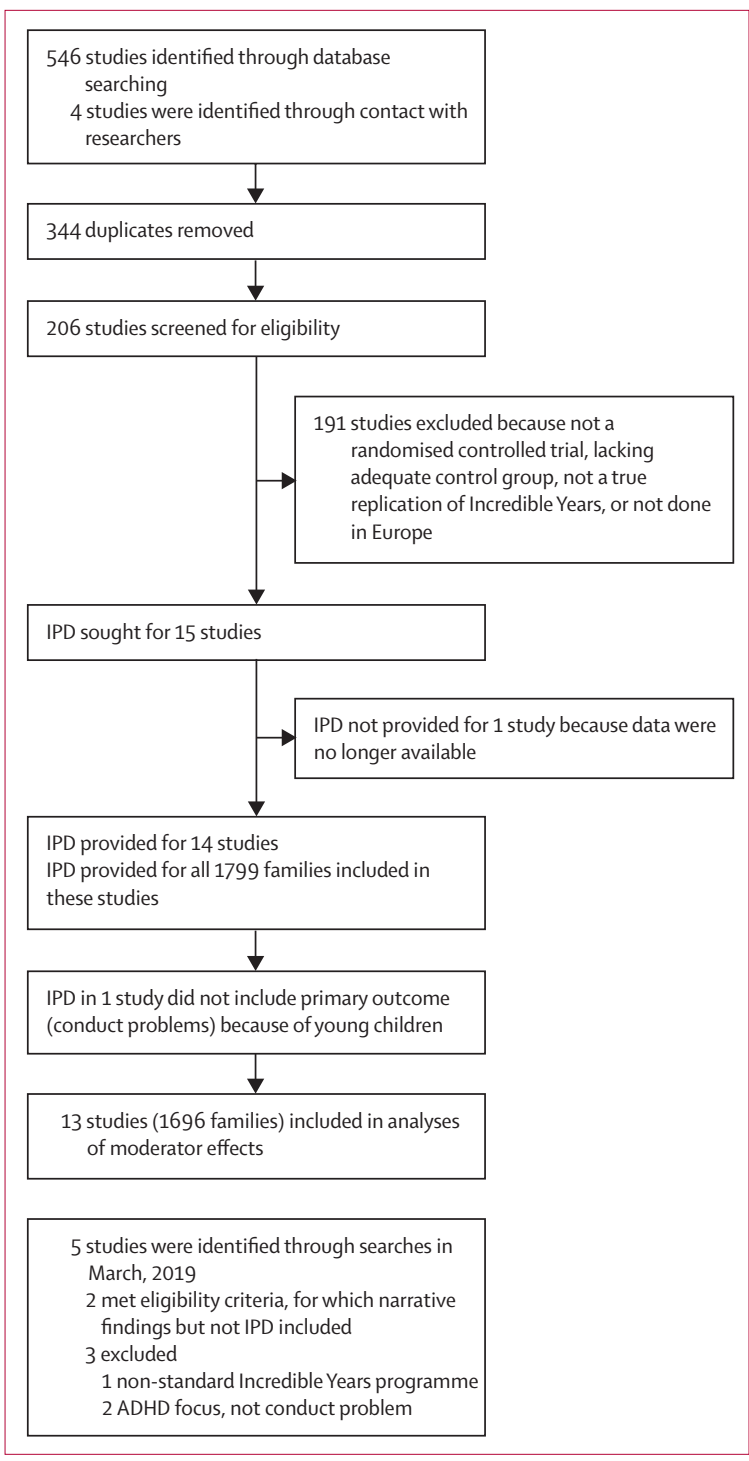

Figure 1: Study selection

$\mathrm{IPD}=$ individual participant data.

primary parent was aged younger than 20 years at the target child's birth. Joblessness was indicated by there being no parent in the household who was employed. Ethnic minority status was indicated by whether the primary parent's ethnic background was any category other than white (Office for National Statistics classification).

Power calculations for an anticipated sample size of 1400 gave $96 \%$ (Cohen's $d=0 \cdot 20)$ and $80 \%(0 \cdot 15)$ power to detect a small interaction effect between two binary variables using ANOVA F-test at 5\% significance level.

We aimed to assess whether any of the six binary, participant-level measures of social disadvantage moderated the effect of Incredible Years on ECBI-I score, post-intervention. Three statistical issues needed addressing. First, the pooled data had a hierarchical 


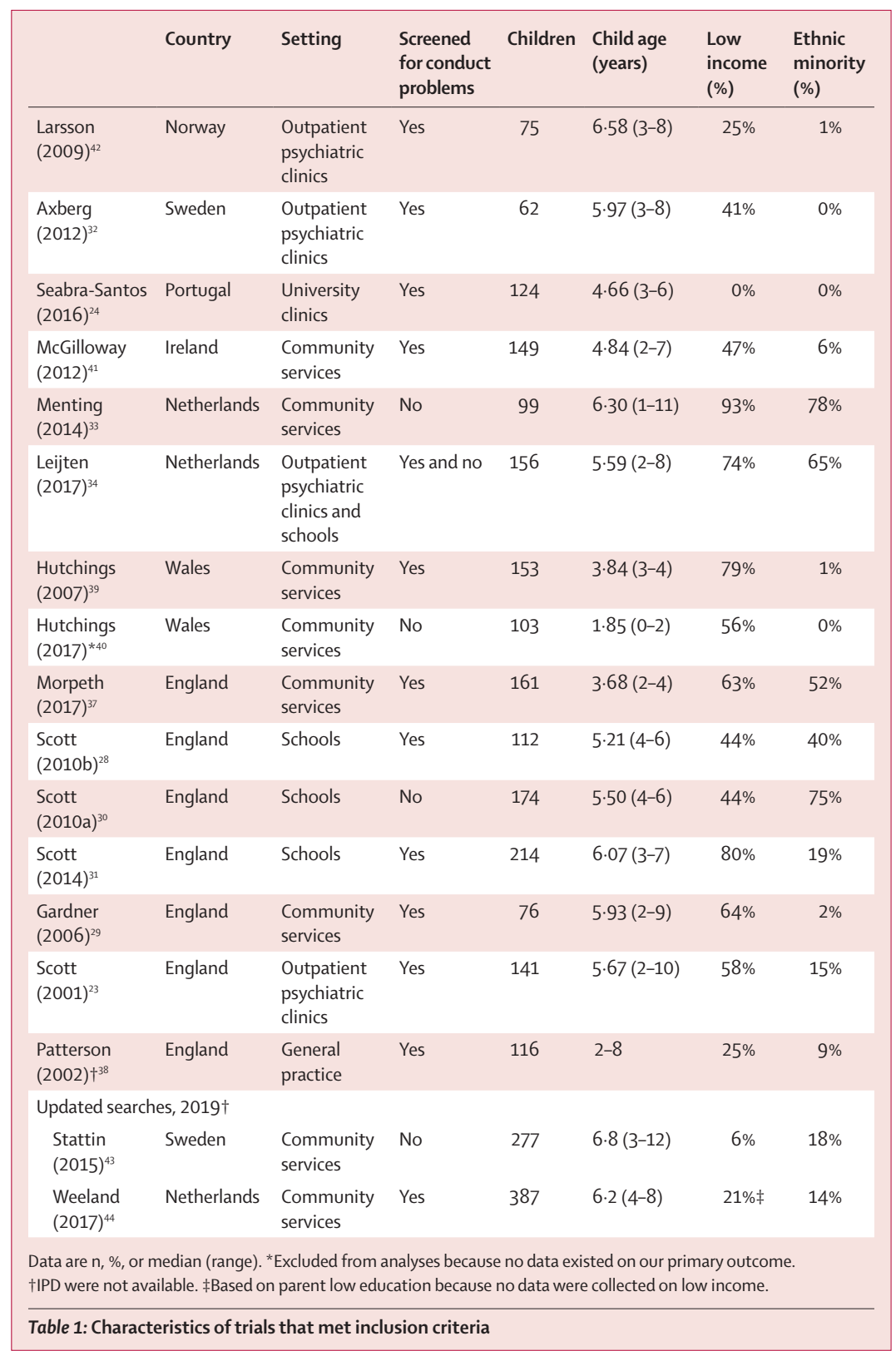

structure, in which families (level 1) in the intervention group were nested within parent groups (level 2), and parent groups were nested within trials (level 3). Second, there was some variation in design of the trials that needed accounting for (eg, stratified randomisation and changes in allocation ratios during the trial). Third, missing data biases needed minimising. We addressed these issues using a one-stage model, which tackles relevant moderation questions, all in one step. ${ }^{20}$

Putative moderators were assessed one at a time. The resulting multilevel modelling used the post-intervention ECBI-I score as the dependent variable and contained fixed effects for trial group, trial-level moderator summaries (between-trial variables, eg, percentage of ethnic minority children), participant-level deviations from trial summaries (within-trial variables), and respective interaction terms. Tests of the effects of interaction terms provided an assessment of the trial-level and participant-level moderating effects. Importantly, this method allowed us to assess empirically whether these two moderating effects differed. If such a difference was significant at a liberal $10 \%$ test level, then two separate moderating effects were allowed; if not, a more powerful model with a single interaction term was fitted. The sizes of any moderation effects were described by an effect moderation index that expressed the difference in intervention effect (active minus control arm) between presence and absence of the binary moderator on the ECBI-I scale.

The hierarchical structure of the data was modelled by random intercepts that varied with trial (level 3) and a further random intercept that varied with parent training group within the active trial arm (level 2). Trial design features were accommodated by including relevant fixed effects (eg, for randomisation stratifiers) or random intercepts that varied with cluster in a cluster-randomised trial. Known predictors of post ECBI-I score (baseline ECBI-I score, child sex, or age) were also included as fixed effects, as was the possible confounderprevention versus treatment trial-and its interaction with trial group, to adjust moderation effects. Finally, to allow for further treatment effect heterogeneity (eg, due to service contexts or composition of the trial population) a trial-varying random coefficient of trial arm was included in the model.

IPD had missing values in moderator and outcome variables. We used multiple imputation by chained equations $^{36}$ to produce valid estimates of moderation effects under missing-at-random assumptions. ${ }^{36}$ Analyses were done in Stata (version 14). The significance level was $5 \%$, unless specified.

\section{Role of the funding source}

The funder of the study had no role in study design, data collection, data analysis, data interpretation, or writing of the report. The corresponding author had full access to all the data in the study and had final responsibility for the decision to submit for publication.

\section{Results}

206 studies were screened for eligibility. 15 trials met the inclusion criteria (figure 1, table 1), done in England $(\mathrm{k}=7),{ }^{23,28-31,37,38}$ Wales $(\mathrm{k}=2),{ }^{39,40}$ Netherlands $(\mathrm{n}=2),{ }^{33,34}$ and one each in Ireland, ${ }^{41}$ Norway, ${ }^{42}$ Portugal ${ }^{24}$ and Sweden. ${ }^{32}$ $13(87 \%)$ trials $(n=1696)$ were included in the analyses, with two (13\%) UK trials excluded, one in which data were no longer available ${ }^{38}$ and one in which IPD were supplied but did not assess data on our primary outcome, ${ }^{40}$ because of the young age of the children. Because of uneven (2:1) randomisation ratios in some trials, there were 1046 families in the intervention arms and 650 in the 
control arms. For all trials, we included data for baseline and first post-intervention assessment, which was normally 4-6 months after the intervention. In most studies the first post-intervention assessment was the primary endpoint. All trials were done independently of the US-based developer (Webster-Stratton). Risk of bias within studies was assessed as low on most items (appendix).

PACS and ECBI-I scores were strongly positively correlated $(\mathrm{r}=0 \cdot 71)$, based on IPD from four trials $\mathrm{s}^{28-31}$ that included both measures. Internal consistency at baseline was high (ECBI-I $\alpha=0.94$; PACS $\alpha=0 \cdot 82$ ). Data from primary caregivers ( $98 \%$ mothers) were used because few trials included data from both parents. Few data $(\mathrm{k}=3)$ were available from other informants (eg, teachers) and were excluded.

Of the 13 trials included in the IPD analyses, ten were studies of treatment (five referred for clinical-level conduct problems) or indicated prevention (five screened for high levels of conduct problems). Three were selective prevention trials (targeting high-risk families such as disadvantaged families or mothers who had been released from prison). ${ }^{30,33,34}$ Some trials targeted lowincome areas (Wales Sure Start) ${ }^{39}$ or schools in lowincome areas $^{28}$ before screening for conduct problems. Overall, most trials (ten of 13 [77\%]) included families who were predominantly socially disadvantaged because of low income or lone parenthood. . $33,28-31,33,34,37,39,41$ Six (46\%) trials in England and the Netherlands accounted for over $90 \%$ of the families from ethnic minorities (range 19-78\% per trial). ${ }^{28,30,31,33,34,37}$ In nine trials, the control condition was a waiting list and in four trials there was minimal or no intervention. Most sites (nine of 13 [69\%]) delivered Incredible Years in community settings (eg, schools, family centres, non-governmental organisations), rather than health services (three of 13 [23\%]).

More than half the families (931/1614 [58\%]) had low income, 35\% [57/1606] were lone parents, or jobless [45/1303]; 492 (30\%) of 1651 were from ethnic minorities (table 2). In one Dutch trial, families identified as mainly Middle Eastern and North African; in one Dutch and three London, UK, trials, mainly families identified as African-Caribbean, alongside multiple other ethnic groups. In Birmingham, families identified as belonging to 18 different ethnic groups. The mean child age was 5 years (63 months, SD 17.8). 326 (23\%) of 1393 parents reported clinical levels of depressive symptoms. Families in the trial with no IPD available ${ }^{38}(n=116)$ were moderately similar to those in other trials. Children aged 2-8 years from three English general practices were screened for above-average conduct problems. Families were less disadvantaged than were those in the pool average, and $11(9 \%)$ of 115 children were ethnic minority. Updated searches in 2019 found two further eligible trials, not included in the IPD analysis, in Sweden $^{43}$ and the Netherlands. ${ }^{44}$ Both were done in community settings and were aimed at children who showed heightened

\begin{tabular}{|c|c|c|c|c|}
\hline & Total & $\begin{array}{l}\text { Trials } \\
\text { available }\end{array}$ & $\begin{array}{l}\text { Control } \\
(\mathrm{N}=650)\end{array}$ & $\begin{array}{l}\text { Incredible } \\
\text { Years } \\
(\mathrm{N}=1046)\end{array}$ \\
\hline \multicolumn{5}{|l|}{ Categorical variable } \\
\hline Child sex (male) & 1696 & 13 & $650(63 \cdot 8 \%)$ & $1046(63 \cdot 1 \%)$ \\
\hline Low income & 1614 & 13 & $615(57 \cdot 9 \%)$ & $999(57 \cdot 6 \%)$ \\
\hline Low education & 1561 & 13 & $581(39 \cdot 8 \%)$ & $980(43 \cdot 3 \%)$ \\
\hline Lone parent & 1606 & 13 & $606(33 \cdot 0 \%)$ & $1000(36 \cdot 8 \%)$ \\
\hline Teenage parent & 1609 & 12 & $605(12 \cdot 6 \%)$ & $1004(11 \cdot 7 \%)$ \\
\hline Jobless household & 1303 & 11 & $522(30 \cdot 3 \%)$ & $781(37 \cdot 5 \%)$ \\
\hline Ethnic minority & 1651 & 13 & $629(30 \cdot 0 \%)$ & $1022(30 \cdot 9 \%)$ \\
\hline \multicolumn{5}{|l|}{ Continuous variable } \\
\hline ECBI-I (baseline) & 1622 & 13 & $\begin{array}{l}611 \\
(135 \cdot 5[37 \cdot 0])\end{array}$ & $\begin{array}{l}1011 \\
(139 \cdot 4[37 \cdot 0])\end{array}$ \\
\hline $\begin{array}{l}\text { ECBI-I (post } \\
\text { intervention) }\end{array}$ & 1445 & 13 & $\begin{array}{l}567 \\
(125 \cdot 5[37 \cdot 9])\end{array}$ & $\begin{array}{l}878 \\
(116 \cdot 2[34 \cdot 7])\end{array}$ \\
\hline Child age (months) & 1682 & 13 & $\begin{array}{l}643 \\
(64 \cdot 2[16 \cdot 9])\end{array}$ & $\begin{array}{l}1039 \\
(62 \cdot 4[18 \cdot 3])\end{array}$ \\
\hline \multicolumn{5}{|c|}{$\begin{array}{l}\text { Data are } \mathrm{n}, \mathrm{n}(\%) \text {, or } \mathrm{n} \text { (mean [SD]). ECBI-I=Eyberg Child Behavior Inventory } \\
\text { Intensity. }\end{array}$} \\
\hline
\end{tabular}

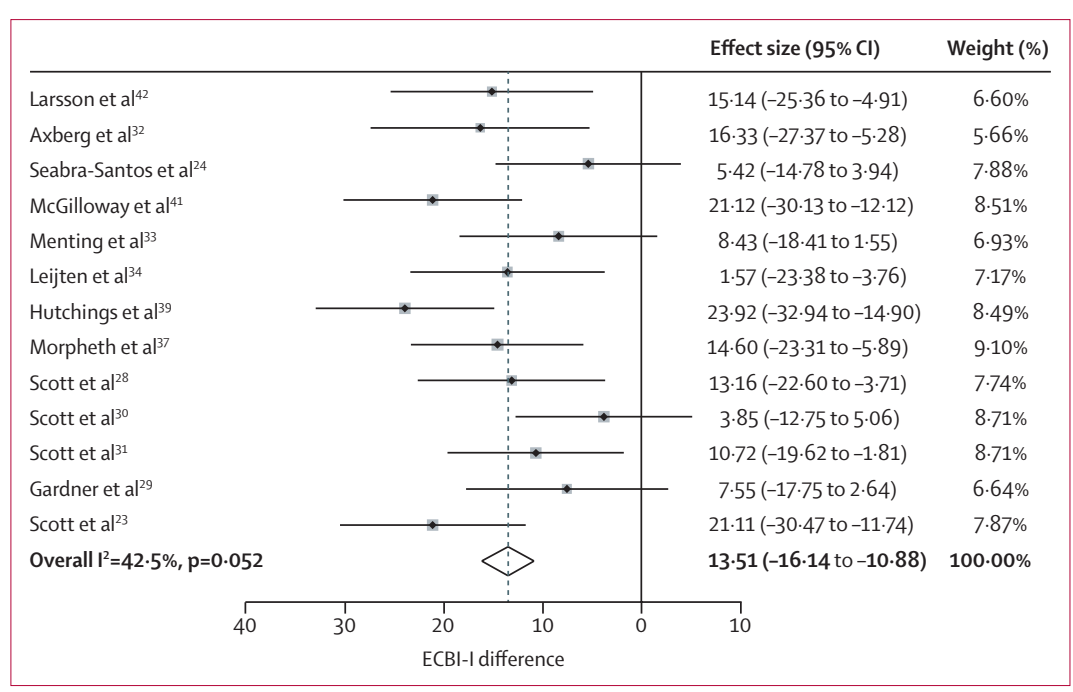

Figure 2: Estimated effects of Incredible Years on child conduct problems

Symptom reductions indicate a benefit of Incredible Years. Effects were estimated based on the individual participant data by fitting the simplified analysis model to each trial separately. ECBI-I=Eyberg Child Behavior Inventory Intensity.

conduct problems. Percentages of families with low income and who were from an ethnic minority were somewhat lower than they were in the sample average (table 1).

There was a significant overall effect of Incredible Years intervention on child conduct problems $(\mathrm{z}=10 \cdot 08$, $\mathrm{p}<0 \cdot 001)$, estimated to be a reduction of $13 \cdot 5$ points on ECBI-I (95\% CI 10·9-16·1). Our sample mean ECBI-I score at baseline was 137 (SD 37) with post-test mean in the intervention groups of 116 (SD 35), and 125 in the control group (possible range 35-252). The clinical cutoff for conduct problems is 127; thus, intervention group 


\begin{tabular}{|c|c|c|c|c|}
\hline & $\begin{array}{l}\text { Percentage } \\
\text { with modifier }\end{array}$ & $\begin{array}{l}\text { Estimated* }^{*} \\
\text { intervention effect } \\
\text { (ECBI-I score) }\end{array}$ & $\begin{array}{l}\text { Estimated moderation } \\
\text { index }(95 \% \mathrm{Cl})\end{array}$ & $p$ value \\
\hline \multicolumn{5}{|l|}{ Income } \\
\hline Low income & $58 \%$ & $-11 \cdot 52$ & $1.91(-4.77$ to 8.59$)$ & 0.58 \\
\hline Not low income & $42 \%$ & $-13 \cdot 43$ & .. & .. \\
\hline \multicolumn{5}{|l|}{ Education } \\
\hline Low education & $39 \%$ & $-9 \cdot 88$ & $4 \cdot 37(-2 \cdot 17$ to $10 \cdot 90)$ & 0.29 \\
\hline Not low education & $61 \%$ & $-14 \cdot 25$ & .. & .. \\
\hline \multicolumn{5}{|c|}{ Lone parenthood status } \\
\hline Lone parent & $35 \%$ & $-12 \cdot 57$ & $0 \cdot 5(-6 \cdot 1$ to $7 \cdot 1)$ & 0.88 \\
\hline Not lone parent & $65 \%$ & $-13 \cdot 07$ &.. & .. \\
\hline \multicolumn{5}{|c|}{ Teenage parenthood status } \\
\hline Teenage parent & $12 \%$ & $-6 \cdot 36$ & $7 \cdot 32(-2 \cdot 24$ to $16 \cdot 87) \dagger$ & $0.13 \dagger$ \\
\hline Not teenage parent & $88 \%$ & $-13 \cdot 68$ & .. & .. \\
\hline \multicolumn{5}{|l|}{ Employment } \\
\hline Jobless household & $34 \%$ & $-8 \cdot 17$ & $4.88(-2.67$ to 12.42$)$ & 0.21 \\
\hline Employed & $66 \%$ & -13.05 & .. & .. \\
\hline \multicolumn{5}{|l|}{ Ethnicity } \\
\hline Ethnic minority & $30 \%$ & $-14 \cdot 96$ & $-1 \cdot 37(-9.81$ to $7 \cdot 08) \dagger$ & $0.75 \dagger$ \\
\hline Not ethnic minority & $70 \%$ & $-13 \cdot 59$ & .. & .. \\
\hline
\end{tabular}

ECBI-I=Eyberg Child Behavior Inventory Intensity. *Moderators were modelled one at a time and thus adjustments for missing data biases can vary between models. †Separate interaction terms fitted for between-trial and within-trial effects. Reported inferences refer to within-trial moderation effects.

Table 3: Formal assessments of within-trial moderators

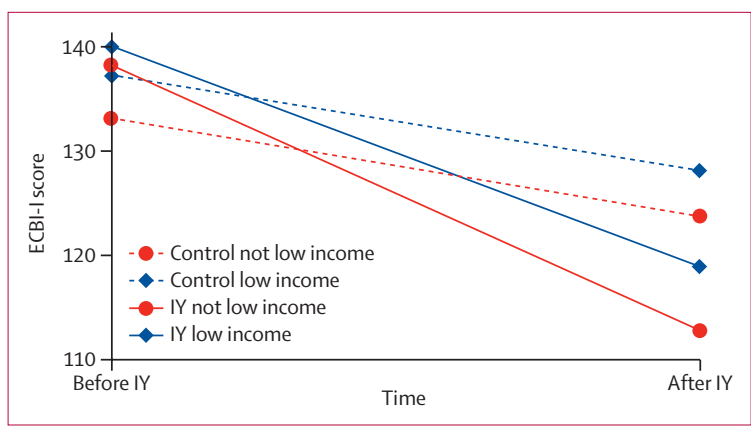

Figure 3: Moderation of intervention effects on child conduct problems by low income

Scores are unadjusted raw mean ECBI-I scores ( $n=1614)$. ECBI-I=Eyberg Child Behavior Inventory Intensity. IY=Incredible Years.

children on average moved from 10 points above to 10 points below the cutoff and children in the control group moved to near the cutoff after testing. Table 2 summarises ECBI-I data from before and after testing by trial arm. Figure 2 shows trial-specific and overall intervention effects. Most trials found that Incredible Years reduced conduct problems. Standardised group differences varied from very small $(-0 \cdot 10$, equivalent to a 3.9 ECBI-I point reduction based on baseline ECBI-I SD of 37 points) to moderate or large $(-0.65 ; 23.9$ points $)$. Our overall effect was small-to-moderate sized $(-0 \cdot 37$, $95 \% \mathrm{CI}-0 \cdot 44$ to $-0 \cdot 29 ; 13 \cdot 5$ points decrease). As found in aggregate-level reviews, ${ }^{3}$ indicated prevention or treatment trials had larger effect sizes $^{32,39,42}$ than did selective prevention trials. ${ }^{30,33,34}$ Between-trial heterogeneity in intervention effects was moderate $\left(I^{2}=42 \cdot 5 \%\right)$.

Five binary variables (low income, low education, joblessness, lone parent, and teenage parent) were used to index aspects of social disadvantage. These variables displayed moderate-to-large positive associations in our sample $(r=0 \cdot 16$ to $0 \cdot 54$; the strongest correlation was between joblessness and low income). Membership of an ethnic minority was not consistently associated with these indices $(r=-0.12$ to 0.07$)$.

Analyses of interaction effects of participant-level variables showed that, overall, there were no significant moderation effects by any social disadvantage indicator, or by ethnicity (table 3 ). Moderation effects at the participant level did not usually differ from those at the trial level (all $\mathrm{p}$ values $>0 \cdot 27$ ), and therefore, we did a moderation assessment across trials and participants. Exceptions were teenage parent status $(\mathrm{p}=0.051)$ and ethnic minority $(\mathrm{p}=0 \cdot 042)$, for which moderation effects differed between and within trials at the $10 \%$ level. Therefore, we report the within-trial results for these variables. None of the relevant moderation tests were significant (table 3). Figure 3 shows raw data means by trial group and the disadvantage indicator, low income. Table 3 shows estimated effect modification indices for each moderator. Even the largest estimated index $(7.3$ points) would separate the overall effect $(\mathrm{d}=-0 \cdot 37)$ into two moderator-present and moderator-absent effects in the same direction $(d=-0 \cdot 47$; $\mathrm{d}=-0 \cdot 27)$. Thus, as well as these moderation indices being statistically insignificant, estimates of their magnitudes are mostly small and unlikely to be clinically important. Notably, both trials retrieved from updated searches reported within-trial moderator analyses, ${ }^{43,44}$ finding no differential effects by social disadvantage indicators, including lone parent, low education, low income, and immigrant status.

\section{Discussion}

This study is the first to use the power of IPD metaanalysis to assess the equity effects of one of the highest quality parenting programmes for reducing conduct problems, a common and disabling childhood mental health condition. Conduct problems are several times more common in disadvantaged groups, ${ }^{7}$ and, if untreated, can lead to a high prevalence of criminality, poor mental health, and poor social functioning, such as poverty and unemployment. ${ }^{2}$ It would be a matter of grave concern if parenting interventions further widened the differences between socioeconomic groups, thereby contributing to an inequitable society. Disadvantaged families would plausibly derive less benefit than advantaged families, since parenting interventions require social circumstances that allow sufficient time and organisation to implement the required behavioural change. Such interventions have been suggested to be particularly susceptible to adverse equity effects. ${ }^{9}$ Our careful synthesis of data from almost all trials of the 
Incredible Years parenting intervention in Europe provided a uniquely large and diverse sample that allowed us to address equity effects using the most stringent and well powered tests to date.

Our findings did not provide any evidence to suggest that families with social disadvantage and those from ethnic minorities benefit less from Incredible Years than do more advantaged families. For most variables, our study was powered to detect even a small moderation effect $(d=0 \cdot 2$, which is a true moderation index of 7.4 ECBI-I points). Thus, the intervention is highly unlikely to have increased existing social inequalities with respect to the amelioration of child conduct problems. Moreover, the programme might reduce social inequity over the long term, if the effects are maintained over time. To date, the only long-term follow-up of European Incredible Years trials supported this hypothesis, with a halving in the rate of oppositional defiant disorder 8-10 years after initial treatment. ${ }^{45}$

Incredible Years has features that might enhance its effectiveness across social groups. Although the programme's content and principles are similar to those of other evidence-based parenting programmes derived from social learning theory (eg, Triple $\mathrm{P}$ and Parent Management Training-Oregon [PMTO]), ${ }^{46,47}$ it has a particular focus on a collaborative delivery model. Thus, parenting goals and strategies are tailored to families' needs, whether these needs arise from child characteristics, culture, social disadvantage, or family values. ${ }^{48}$ This careful attention to individual needs might help to enhance intervention effectiveness in a variety of families, offsetting differential effects that might otherwise result from programmes based on educational and behavioural change.

This study is notable in providing a sophisticated analysis of equity effects by ethnicity in Europe. These are potentially generalisable findings, based on the inclusion of substantial numbers of families from diverse ethnic groups, with different cultural and immigration backgrounds, albeit mainly from six trials in two countries, the UK and the Netherlands. Because of small numbers in most categories of ethnicity, we were unable to look further at specific groups. The intervention was delivered in groups with mixed ethnicities, and rather than being specifically culturally adapted, is collaborative and flexible in its approach. This fact suggests that despite variation in parenting styles and values across ethnicities, Incredible Years does not need adaptation for separate ethnic groups. This finding is important in view of the additional practical and financial demands of service adaptation and delivery that is specific to ethnicity. Moreover, separating delivery systems for different ethnic groups is not beneficial for community integration. The absence of any differential effect by ethnicity might be a feature of Incredible Years' built-in flexibility and cultural sensitivity. ${ }^{48}$ However, further assessment is needed to test whether other parenting programmes show comparable equity effects by socioeconomic status or ethnicity. ${ }^{10,46}$ Additionally, the effects of key delivery components that are relevant to equity (including Incredible Years collaborative approach, which can be costly to train) require further investigation using factorial designs to optimise effectiveness.

Our study has several further strengths that increase our confidence in the findings. First, our unique pooled dataset yielded a large sample and results, which, because of the diversity of samples and contexts, are potentially generalisable across countries, service settings, and severity of child problems. Second, compared with other IPD studies, we obtained an unusually complete set of data from $93 \%(14 / 15)$ of eligible trials, thereby minimising the possibility of availability bias. One trial in toddlers ${ }^{40}$ supplied IPD but was excluded as it did not assess conduct problem outcomes. Although our original searches were done in 2015, we updated the searches in 2019, finding two trials whose results were consistent with our IPD analyses. Both showed no moderation by social disadvantage, ${ }^{43,44}$ suggesting that their inclusion would be unlikely to alter the conclusions. Third, the plausibility of the findings is enhanced by our robust analytical strategies. We included only randomised trials and accounted for all relevant trial design features. Finally, we accounted for missing data using multiple imputation, which requires less restrictive assumptions than does using completely observed cases. ${ }^{36}$

Our study has several limitations. First, we focused on only one parenting programme, albeit one of the most established in Europe, and whose content and delivery are in many ways similar to other evidence-based parenting programmes. Second, we focused only on trials in Europe to better understand the effects of a programme commonly imported from the USA in the region and because of the availability of a near-complete sample of trials done independently of the commercial developer. Third, we made several assumptions during data harmonisation, notably that two different instruments measured the same construct of conduct problems, and that indices of low socioeconomic status (eg, receipt of benefits) were broadly similar across countries. Fourth, data were available only on the variation in intervention effectiveness. Trials did not assess data on variation in access to parenting services, another potential source of inequalities. Nevertheless, most trials appeared successful in accessing disadvantaged families because families of low-socioeconomic status and lone-parent families were over-represented, compared with national norms..$^{23,28-31,37,39,40}$ Fifth, insufficient data were available from other informants (eg, fathers, teachers); hence, we could include only data from one parent, usually the mother, as a primary outcome. Parent-reported conduct problems (typically by the mother) is the usual primary outcome for trials in this age group. As with any pooled data study, we were able to include only those outcomes that were measured in individual trials. Direct observational measures of conduct problems provide one solution for 
validating self-reported outcomes; ${ }^{21}$ however, these were highly heterogeneous and available only in a subset of trials. Future trials should involve multiple data sources, using common instruments across the field.

Our pooled IPD meta-analysis, the largest on any intervention for conduct problems or parenting, suggests that Incredible Years does not increase socioeconomic inequalities in families with children with child conduct problems. This finding contrasts with earlier findings based on aggregate-level analyses and inferior trial designs, ${ }^{12,13}$ which might have been underpowered and subject to ecological fallacy. Families from disadvantaged and advantaged backgrounds, and those from ethnic minorities, are all likely to benefit from the intervention. This conclusion probably applies to other parenting interventions with similar content and collaborative delivery, although we cannot be certain without further IPD data. For example, a small Norwegian study combined two trials of another US programme, PMTO, and found no adverse equity effects. ${ }^{46}$ Our findings are important for policymakers and commissioners needing to identify, fund, and target interventions for those who are most at risk, as well as practitioners recommending or delivering parenting programmes. The Incredible Years intervention is clearly an effective strategy to reduce conduct problems in families facing social disadvantage. However, to avoid adverse equity effects, services must continue to ensure that the most disadvantaged families can access the programme.

\section{Contributors}

FG was principal investigator and drafted the paper. FG, JH, and SS conceived the study. FG, SS, SL, JH, and JB designed the study, wrote the grant, and interpreted the findings. SL designed the analyses and SL and VH did the analyses. PL managed the study and data, contributed to the design and writing, and interpreted the findings, assisted by JM. FG, PL, JH, SS, VB, SM, MG, MJSS, BOdC, AM, UA, and WM led included trials and contributed trial data and to the interpretation of findings. MW and EMB contributed to the interpretation of the findings. All authors commented upon and approved the final manuscript.

\section{Declaration of interests}

FG, PL, JH, SS, VB, SM, MG, MJSS, BOdC, AM, UA, and WM led trials that were included in the individual patient dataset. JH, MG, and MJSS report occasional payments for training leaders in the Incredible Years parent programme. FG and $\mathrm{JH}$ are co-developers of a non-profit parenting programme with WHO, Parenting for Lifelong Health. All other authors declare no other competing interests.

\section{Acknowledgments}

The National Institute for Health Research (NIHR) Public Health Research funded the study (grant 12-3070-04, PI FG). This research represents independent research, with SL part funded by the NIHR Biomedical Research Centre at South London and Maudsley NHS Foundation Trust and King's College London, London, UK. VB is supported by the NIHR Collaboration for Leadership in Applied Health Research and Care South West Peninsula. The views expressed are those of the authors and not necessarily those of the NHS, the NIHR, or the UK Department of Health.

\section{References}

1 Erskine HE, Norman RE, Ferrari AJ, et al. Long-term outcomes of attention-deficit/hyperactivity disorder and conduct disorder: a systematic review and meta-analysis.

J Am Acad Child Adolesc Psychiatry 2016; 55: 841-50.
2 Rivenbark JG, Odgers CL, Caspi A, et al. The high societal costs of childhood conduct problems: evidence from administrative records up to age 38 in a longitudinal birth cohort. J Child Psychol Psychiatry 2018; 59: 703-10.

3 Leijten P, Gardner F, Melendez-Torres GJ, et al. Meta-analyses: key parenting program components for disruptive child behavior. J Am Acad Child Adolesc Psychiatry 2019; 58: 180-90.

4 van Aar J, Leijten P, Orobio de Castro B, Overbeek G. Sustained, fade-out or sleeper effects? A systematic review and meta-analysis of parenting interventions for disruptive child behavior. Clin Psychol Rev 2017; 51: 153-63.

5 WHO. Inspire: seven strategies for ending violence against children. World Health Organization, Geneva; 2016.

6 Scott S. A national approach to improving child and adolescent mental health care: the children and young people's improving access to psychological therapies (IAPT) programme in England. In: Weisz JR, Kazdin AE, eds. Evidence-based psychotherapies for children and adolescents. New York: Guilford Press, 2017: 415-28.

7 Piotrowska PI, Stride CB, Maughan B, Goodman R, McCaw L, Rowe R. Income gradients within child and adolescent antisocial behaviours. Br J Psychiatry 2015; 207: 385-91.

8 Kouider EB, Koglin U, Petermann F. Emotional and behavioral problems in migrant children and adolescents in Europe: a systematic review. Eur Child Adolesc Psychiatry 2014; 23: 373-91.

9 Lorenc T, Petticrew M, Welch V, Tugwell P. What types of interventions generate inequalities? Evidence from systematic reviews. J Epidemiol Community Health 2013; 67: 190-93.

10 Gardner F, Connell A, Trentacosta CJ, Shaw DS, Dishion TJ, Wilson MN. Moderators of outcome in a brief family-centered intervention for preventing early problem behavior. J Consult Clin Psychol 2009; 77: 543-53.

11 Leijten P, Raaijmakers MAJ, de Castro BO, Matthys W. Does socioeconomic status matter? A meta-analysis on parent training effectiveness for disruptive child behavior. J Clin Child Adolesc Psychol 2013; 42: 384-92.

12 Lundahl B, Risser HJ, Lovejoy MC. A meta-analysis of parent training: moderators and follow-up effects. Clin Psychol Rev 2006; 26: 86-104.

13 Reyno SM, McGrath PJ. Predictors of parent training efficacy for child externalizing behavior problems-a meta-analytic review. J Child Psychol Psychiatry 2006; 47: 99-111.

14 Belsky J, Melhuish E, Barnes J, Leyland AH, Romaniuk H. Effects of Sure Start local programmes on children and families: early findings from a quasi-experimental, cross sectional study. BMJ 2006; 332: 1476

15 Gardner F, Montgomery P, Knerr W. Transporting evidence-based parenting programs for child problem behavior (age 3-10) between countries: systematic review and meta-analysis. J Clin Child Adolesc Psychol 2016; 45: 749-62.

16 Reid M, Webster-Stratton C, Beauchaine TP. Parent training in Head Start: a comparison of program response among African American, Asian American, Caucasian, and Hispanic Mothers. Prev Sci 2001; 2: 209-27.

17 van Mourik K, Crone MR, de Wolff MS, Reis R. Parent training programs for ethnic minorities: a meta-analysis of adaptations and effect. Prev Sci 2017; 18: 95-105.

18 Castro FG, Barrera M, Holleran Steiker LK. Issues and challenges in the design of culturally adapted evidence-based interventions. Annu Rev Clin Psychol 2010; 6: 213-39.

19 Petticrew M, Tugwell P, Kristjansson E, Oliver S, Ueffing E, Welch V. Damned if you do, damned if you don't: subgroup analysis and equity. J Epidemiol Community Health 2012; 66: 95-98.

20 Fisher DI, Carpenter JR, Morris TP, Freeman SC, Tierney JF. Meta-analytical methods to identify who benefits most from treatments: daft, deluded, or deft approach? BMJ 2017; 356: j573.

21 Menting AT, Orobio de Castro B, Matthys W. Effectiveness of the Incredible Years parent training to modify disruptive and prosocial child behavior: a meta-analytic review. Clin Psychol Rev 2013; 33: 901-13.

22 Eyberg SM, Ross AW. Assessment of child behavior problems: the validation of a new inventory. J Clin Child Psychol 1978; 7: 113-16.

23 Scott S, Spender Q, Doolan M, Jacobs B, Aspland H. Multicentre controlled trial of parenting groups for childhood antisocial behaviour in clinical practice. BMJ 2001; 323: 194-98. 
24 Seabra-Santos MJ, Gaspar MF, Azevedo AF, et al. Incredible Years parent training: what changes, for whom, how, for how long? J Appl Dev Psychol 2016; 44: 93-104.

25 Taylor E, Schachar R, Thorley G, Wieselberg M. Conduct disorder and hyperactivity: I. Separation of hyperactivity and antisocial conduct in British child psychiatric patients. Br J Psychiatry 1986; 149: 760-67.

26 Taylor E, Sandberg S, Thorley G. The epidemiology of childhood hyperactivity (vol 33). Oxford: Oxford University Press, 1991

27 Burns GL, Patterson DR. Normative data on the Eyberg Child Behavior Inventory and Sutter-Eyberg Student Behavior Inventory: parent and teacher rating scales of disruptive behavior problems in children and adolescents. Child Fam Behav Ther 2001; 23: 15-28.

28 Scott S, Sylva K, Doolan M, et al. Randomised controlled trial of parent groups for child antisocial behaviour targeting multiple risk factors: the SPOKES project. J Child Psychol Psychiatry 2010b; 51: 48-57.

29 Gardner F, Burton J, Klimes I. Randomised controlled trial of a parenting intervention in the voluntary sector for reducing child conduct problems: outcomes and mechanisms of change. J Child Psychol Psychiatry 2006; 47: 1123-32.

30 Scott S, O'Connor TG, Futh A, Matias C, Price J, Doolan M. Impact of a parenting program in a high-risk, multi-ethnic community: the PALS trial. J Child Psychol Psychiatry 2010a; 51: 1331-41.

31 Scott S, Sylva K, Kallitsoglou A, Ford T. Which type of parenting programme best improves child behaviour and reading? Follow-up of the Helping Children Achieve trial. London: Nuffield Foundation, 2014.

32 Axberg U, Broberg AG. The Incredible Years: evaluation of the Webster Stratton parent management training programme in Sweden. Scand J Psychol 2012; 53: 224-32.

33 Menting ATA, de Castro BO, Wijngaards-de Meij LDNV, Matthys W. A trial of parent training for mothers being released from incarceration and their children. J Clin Child Adolesc Psychol 2014; 43: 381-96

34 Leijten P, Raaijmakers MA, Orobio de Castro B, van den Ban E, Matthys W. Effectiveness of the Incredible Years parenting program for families with socioeconomically disadvantaged and ethnic minority backgrounds. J Clin Child Adolesc Psychol 2017; 46: 59-73.

35 UNESCO. International standard classification of education 2011. Montreal: UNESCO Institute for Statistics, 2011.

36 White IR, Royston P, Wood AM. Multiple imputation using chained equations: issues and guidance for practice. Stat Med 2011; 30: $377-99$.
37 Morpeth L, Blower S, Tobin K, et al. The effectiveness of the Incredible Years pre-school parenting programme in the United Kingdom: a pragmatic randomised controlled trial. Child Care Pract 2017; 23: 141-61.

38 Patterson J, Barlow J, Mockford C, Klimes I, Pyper C, Stewart-Brown S. Improving mental health through parenting programmes: block randomised controlled trial. Arch Dis Child 2002; 87: 472-77.

39 Hutchings J, Bywater T, Daley D, et al. Parenting intervention in Sure Start services for children at risk of developing conduct disorder: pragmatic randomised controlled trial. BMJ 2007; 334: 678.

40 Hutchings J, Griffith N, Bywater T, Williams M. Evaluating the Incredible Years toddler parenting programme with parents of toddlers in disadvantaged (Flying Start) areas of Wales. Child Care Health Dev 2017; 43: 104-13.

41 McGilloway S, Ni Mhaille G, Bywater T, et al. A parenting intervention for childhood behavioral problems: a randomized controlled trial in disadvantaged community-based settings. J Consult Clin Psychol 2012; 80: 116-27.

42 Larsson B, Fossum S, Clifford G, Drugli MB, Handegard BH, Morch WT. Treatment of oppositional defiant and conduct problems in young Norwegian children: results of a randomized controlled trial. Eur Child Adolesc Psychiatry 2009; 18: 42-52.

43 Stattin H, Enebrink P, Özdemir M, Giannotta F. A national evaluation of parenting programs in Sweden: the short-term effects using an RCT effectiveness design. J Consult Clin Psychol 2015; 83: 1069-84.

44 Weeland J, Chhangur RR, van der Giessen D, Matthys W, de Castro BO, Overbeek G. Intervention effectiveness of the Incredible Years: new insights into sociodemographic and intervention-based moderators. Behav Ther 2017; 48: 1-18.

45 Scott S, Briskman J, O'Connor TG. Early prevention of antisocial personality: long-term follow-up of two randomized controlled trials comparing indicated and selective approaches. Am J Psychiatry 2014 171: 649-57.

46 Tømmeraas T. Social gradients and participant characteristics in child behavior problem interventions. Child Youth Serv Rev 2016; 70: 57-64.

47 Nowak C, Heinrichs N. A comprehensive meta-analysis of Triple P-Positive Parenting Program using hierarchical linear modeling: effectiveness and moderating variables. Clin Child Family Psychol Rev 2008; 11: 114-25.

48 Webster-Stratton C. Affirming diversity: Multi-cultural collaboration to deliver the incredible years parent programs. Int J Child Health Hum Dev 2009; 2: 17-32. 